\title{
On the Relationship Between Regression Analysis and Mathematical Programming
}

\author{
DONG QIAN WANG† \\ School of Mathematical and Computing Sciences \\ Victoria University of Wellington, NZ \\ STEFANKA CHUKOVA \\ School of Mathematical and Computing Sciences \\ Victoria University of Wellington, $N Z$ \\ C. D. LAI \\ Institute of Information Sciences and Technology \\ Massey University, Palmerston North, NZ
}

\author{
dong.wang@mcs.vuw.ac.nz \\ stefanka.chukova@mcs.vuw.ac.nz \\ c.lai@massey.ac.nz
}

\begin{abstract}
The interaction between linear, quadratic programming and regression analysis are explored by both statistical and operations research methods. Estimation and optimization problems are formulated in two different ways: on one hand linear and quadratic programming problems are formulated and solved by statistical methods, and on the other hand the solution of the linear regression model with constraints makes use of the simplex methods of linear or quadratic programming. Examples are given to illustrate the ideas.
\end{abstract}

Keywords: Regression analysis, linear programming, simplex method, two-phase methods, least squares method, quadratic programming and artificial variable.

\section{Introduction}

We will discuss the interaction between linear, quadratic programming and regression analysis. These interactions are considered both from a statistical point of view and from an optimization point of view. We also examine the algorithms established by both statistical and operations research methods. Minimizing the sum of the absolute values of the regression has shown that it can be reduced to a general linear programming problem (Wager, 1969) and Wolfe (1959) hinted that his method can be applied to regression but no analysis is done. Estimation and optimization problems are formulated in two different ways: on one hand linear and quadratic

$\lceil$ Requests for reprints should be sent to Dong Qian Wang, School of Mathematical and Computing Sciences,Victoria University of Wellington, P.O.Box 600, Wellington, NZ. 
programming problems are formulated and solved by statistical methods, and on the other hand the solution of the linear regression model with constraints makes use of the simplex methods of linear or quadratic programming. Examples are given to show some practical applications of the methods. It is our aim that students taking a linear or non-linear programming (NLP) courses as well as a course in linear models will now realize that there is a definite connection between these problems.

\subsection{Regression models}

Consider the linear regression (LR) model with nonnegative constraints

$$
\begin{aligned}
Y(\beta) & =X \beta+\epsilon \\
\text { subject to } \beta & \geq 0,
\end{aligned}
$$

where $Y \in \mathbf{R}^{n}$ represents the vector of responses, $X$ is an $n \times p$ design matrix, $\beta \in \mathbf{R}^{p}$ represents the unknown parameters of the model and $\beta \geq 0$ means that all the elements in the vector are non-negative, and $\epsilon$ represents the random error term of the LR model.

A general linear regression model (1) with inequality constraints (LRWC) and nonnegative variables is given as follows:

$$
\begin{aligned}
Y(\beta) & =X \beta+\varepsilon \\
\text { subject to } A \beta & \geq C \\
\beta & \geq 0,
\end{aligned}
$$

where $\beta \in \mathbf{R}^{p}$ is the unknown vector; $X_{n \times p}(n \geq p)$ and $A_{s \times p}(s \leq p)$ are constant matrices, $Y_{n \times 1}, C_{s \times 1}$, and $\varepsilon_{n \times 1}$ are column vectors, $\varepsilon \sim N\left(0, \sigma^{2} I\right)$ ; $X^{T} X \geq 0$ and $\operatorname{rank}(A)=s$. The solution of LRWC is a subject of the Karush-Kuhn-Tucker theorem. The subsequent algorithms for solving LRWC are discussed in Lawson and Hanson (1974) and Whittle (1971).

\subsection{Mathematical programming}

Due to the fact that $\max \{f(x)\}=\min \{-f(x)\}$, we will focus our attention only on minimization problems. A primal linear programming (LP) problem with nonnegative solution can be formulated as follows:

$$
\begin{aligned}
\text { minimize } L(\beta) & =b^{T} \beta \\
\text { subject to } G \beta & \geq f \\
\beta & \geq 0,
\end{aligned}
$$


where $\beta$ is the unknown vector, $0 \neq b^{T} \in \mathbf{R}^{p}, f^{T} \in \mathbf{R}^{m}$ and $G_{m \times p}$ are known constant vectors and a matrix, respectively.

A quadratic programming $(\mathrm{QP})$ problem in which all the variables must be nonnegative is formulated as follows:

$$
\begin{aligned}
\text { minimize } Z_{0}(\beta) & =b^{T} \beta+\beta^{T} D \beta \\
\text { subject to } A \beta & \geq C \\
\beta & \geq 0,
\end{aligned}
$$

where $A_{s \times p},(s \leq p), D_{p \times p}$ are matrices; $C_{s \times 1}, \beta_{p \times 1}$ and $b_{p \times 1}$ are column vectors, $\operatorname{rank}(A)=s \leq p$ and $\mathrm{D}$ is symmetric and positive definite matrix.

In the next section, we further explore the relationships between the above four models. The aim in this note is to provide a strong link and algorithms between these concepts. In fact they are equivalent in some cases. Parameter estimates of models (1) and (2) are obtained by the simplex method and the Karush-Kuhn-Tucker theorem. The optimization problems of models (3) and (4) are restated and solved by statistical methods.

\section{Solving Linear Regression Model by Using Mathematical Pro- gramming}

Consider given $n$ observations $\left\{\left(x_{i}, y_{i}\right), i=1,2, \ldots, n\right\}$. The linear regression model (1) with $p=2$, can be rewritten as $Y_{i}=\beta_{0}+\beta_{1} x_{i}+\epsilon_{i}$ and $\beta=\left(\beta_{1}, \beta_{2}\right)^{T} \geq 0$. Using the least squares method to estimate parameters $\beta_{0}$ and $\beta_{1}$, we need to minimize $g(\beta)$, i.e

$$
\begin{array}{ll}
\text { minimize } & g(\beta)=\sum_{i=1}^{n}\left(y_{i}-\beta_{0}-\beta_{1} x_{i}\right)^{2} \\
\text { subject to } & \beta \geq 0 .
\end{array}
$$

The associated system of normal equations is given as follows:

$$
\begin{aligned}
n \beta_{0}+\beta_{1} \sum_{i=1}^{n} x_{i}-\sum_{i=1}^{n} y_{i} & =0, \\
\beta_{0} \sum_{i=1}^{n} x_{i}+\beta_{1} \sum_{i=1}^{n} x_{i}^{2}-\sum_{i=1}^{n} x_{i} y_{i} & =0 .
\end{aligned}
$$

Therefore, model (1) is equivalent to the following mathematical programming problem: 


$$
\begin{array}{ll}
\text { minimize } & g(\beta)=\sum_{i=1}^{n}\left(y_{i}-\beta_{0}-\beta_{1} x_{i}\right)^{2} \\
\text { subject to } & n \beta_{0}+\beta_{1} \sum_{i=1}^{n} x_{i}=\sum_{i=1}^{n} y_{i} \\
& \beta_{0} \sum_{i=1}^{n} x_{i}+\beta_{1} \sum_{i=1}^{n} x_{i}^{2}=\sum_{i=1}^{n} x_{i} y_{i} \\
\beta \geq 0 .
\end{array}
$$

We will use the phase I in the two-phase version of the simplex method to solve (5). The problem to be solved by phase I is

$$
\begin{array}{cl}
\text { maximize } & Y_{0}^{I}=-R_{1}-R_{2} \\
\text { subject to } & n \beta_{0}+c_{1} \beta_{1}+R_{1}=c_{2} \\
& c_{1} \beta_{0}+b_{1} \beta_{1}+R_{2}=b_{2} \\
& \beta \geq 0 \text { and } R_{1}, R_{2} \geq 0 ;
\end{array}
$$

where $R_{1}$ and $R_{2}$ are artificial variables. The optimization problem (6) can be rewritten as

$$
\begin{array}{cl}
\text { maximize } & Y_{0}^{I}=\left(n+c_{1}\right) \beta_{0}+\left(b_{1}+c_{1}\right) \beta_{1}-\left(b_{2}+c_{2}\right) \\
\text { subject to } & n \beta_{0}+c_{1} \beta_{1}+R_{1}=c_{2} \\
& c_{1} \beta_{0}+b_{1} \beta_{1}+R_{2}=b_{2} \\
& \beta_{i} \geq 0 \text { and } R_{i} \geq 0, i=1,2
\end{array}
$$

where $c_{1}=\sum x_{i}, c_{2}=\sum y_{i}, b_{1}=\sum x_{i}^{2}$, and $b_{2}=\sum x_{i} y_{i}$. The initial values are summarized in the Table 1 .

Table 1. The initial values of problem (7).

\begin{tabular}{c|cccc|c}
\hline $\mathrm{BV}$ & $\beta_{0}$ & $\beta_{1}$ & $R_{1}$ & $R_{2}$ & RHS \\
\hline$Y_{0}^{I}$ & $-n-c_{1}$ & $-b_{1}-c_{1}$ & 0 & 0 & $-b_{2}-c_{2}$ \\
\hline$R_{1}$ & $\mathrm{n}$ & $c_{1}$ & 1 & 0 & $c_{2}$ \\
$R_{2}$ & $c_{1}$ & $b_{1}$ & 0 & 1 & $b_{2}$ \\
\hline
\end{tabular}

Bearing in mind that the solution of the LR problem is actually the solution of the corresponding system of normal equations, it is now easy 
to see that problem (7) is equivalent to solving related the LR problem (1). Hence we can obtain the optimal solution for model (1) by using the simplex method for problem (7) with the initial values in Table 1. The above approach can be applied for solving linear regression model (1) with $p>2$, i.e.,

$$
\begin{aligned}
Y_{i}= & \beta_{0}+\beta_{1} x_{i}^{(1)}+\beta_{2} x_{i}^{(2)}+\ldots+\beta_{p} x_{i}^{(p)}+\epsilon_{i} \\
& \text { subject to } \beta \geq 0 .
\end{aligned}
$$

Next, we will illustrate the above ideas by an example.

Example 2.1: (Rencher, 2000, p113-114) The exam scores $y$ and homework scores $x$ (average value) for 18 students in a statistics class were as follows

\begin{tabular}{c|ccccccccc}
$\mathbf{x}$ & 96 & 77 & 0 & 0 & 78 & 64 & 89 & 47 & 90 \\
\hline$y$ & 95 & 80 & 0 & 0 & 79 & 77 & 72 & 66 & 98 \\
\hline $\mathbf{x}$ & 93 & 18 & 86 & 0 & 30 & 59 & 77 & 74 & 67 \\
\hline$y$ & 90 & 0 & 95 & 35 & 50 & 72 & 55 & 75 & 66
\end{tabular}

From the given data set, we obtain: $c_{1}=\sum x_{i}=1,045, c_{2}=\sum y_{i}=1,105$, $b_{2}=\sum x_{i} y_{i}=81,195, b_{1}=\sum x_{i}^{2}=80,199$ and $n=18$. Hence, Table 1 becomes

\begin{tabular}{c|cccc|c|c}
\hline $\mathrm{BV}$ & $\beta_{0}$ & $\beta_{1}$ & $R_{1}$ & $R_{2}$ & RHS & Ratio \\
\hline$Y_{0}^{I}$ & -1063 & -81244 & 0 & 0 & -82300 & - \\
\hline$R_{1}$ & 18 & 1045 & 1 & 0 & 1105 & 1.057 \\
$R_{2}$ & 1045 & 80199 & 0 & 1 & 81195 & 1.012 \\
\hline
\end{tabular}

Using the simplex method, the optimal table is obtained in two iterations as:

\begin{tabular}{c|cc|c|c}
\hline $\mathrm{BV}$ & $\beta_{0}$ & $\beta_{1}$ & RHS & Ratio \\
\hline$Y_{0}^{I}$ & 0 & 0 & 0 & - \\
\hline$\beta_{0}$ & 1 & 0 & 10.727 & - \\
$\beta_{1}$ & 0 & 1 & 0.873 & - \\
\hline
\end{tabular}

Therefore, $\hat{\beta}_{0}=10.727, \hat{\beta}_{1}=0.873$ and a fitted linear regression line is given by $\hat{y}=10.727+0.873 x$. 


\section{Solving the Least Squares Problem With Constraints Using NLP Methods}

Using the least squares method to model (2) we obtain a general regression problem:

$$
\begin{aligned}
\min Z(\beta) & =(Y-X \beta)^{T}(Y-X \beta) \\
\text { subject to } A \beta & \geq C \\
\beta & \geq 0 .
\end{aligned}
$$

Problem (9) is a simultaneous quadratic programming problem and thus it can be solved by using Wolfe's method based on Karush-Kuhn-Tucker conditions. Rewriting problem (9) as a quadratic programming problem leads to:

$$
\begin{aligned}
\min Z_{0}(\beta) & =a+n \beta_{0}^{2}+b_{1} \beta_{1}^{2}-2 c_{2} \beta_{0}-2 b_{2} \beta_{1}+2 c_{1} \beta_{0} \beta_{1} \\
\text { subject to } A \beta & \geq C \\
\beta & \geq 0,
\end{aligned}
$$

where, as in the previous example, $a=\sum y_{i}^{2}, c_{1}=\sum x_{i}, c_{2}=\sum y_{i}$, $b_{2}=\sum x_{i} y_{i}$ and $b_{1}=\sum x_{i}^{2}$.

Example 3.1: Use the given set of data to evaluate the parameters of a simple linear regression model with additional restrictions imposed on the parameters of the model, i.e.,

$$
\begin{aligned}
& 2 \beta_{0}+\beta_{1} \geq 650 \\
& -2 \beta_{0}+\beta_{1} \geq 500 \\
& \beta_{i} \geq 0 \quad i=1,2 . \\
& \begin{array}{c|llllllllll}
\mathbf{x} & .055 & .091 & .138 & .167 & .182 & .211 & .232 & .248 & .284 & .351 \\
\hline y & 90 & 97 & 107 & 124 & 142 & 150 & 172 & 189 & 209 & 253
\end{array}
\end{aligned}
$$

From the given data set,we can calculate the values of $a=259993, c_{1}=$ $\sum x_{i}=1.959, c_{2}=\sum y_{i}=1533, b_{2}=\sum x_{i} y_{i}=341.68 b_{1}=\sum x_{i}^{2}=.4551$ and $n=10$. Parameter estimates of the model obtained by the standard regression technique are $\beta_{0}=39.6484$ and $\beta_{1}=580.151$.

Let us solve the same problem by employing nonlinear programming ideas. Firstly, we have to rewrite the problem in the form of quadratic programming by using the previously calculated values of $b_{1}, b_{2}, c_{1}$ and $c_{2}$. We have: 


$$
\begin{aligned}
\min Z_{0}=259993+10 \beta_{0}^{2}+.4551 \beta_{1}^{2}-3066 \beta_{0}-683.36 \beta_{1}+3.918 \beta_{0} \beta_{1} \\
2 \beta_{0}+\beta_{1} \geq 650 \\
\text { subject to } 2 \beta_{0}+\beta_{1} \geq 500 \\
\beta_{i} \geq 0 \quad i=1,2 .
\end{aligned}
$$

Then, solving the above quadratic programming problem with Wolfe's method confirms the optimal values of $\beta_{0}=39.6484$ and $\beta_{1}=580.151$.

\section{Solving QP Problem Using the Least Squares Method}

The relationship between the quadratic programming (4) and the least squares method (9) is studied by Wang, Chukova and Lai (2003),

Theorem 1: The relationship of QP (4) and LS (9) is given by

$$
Z_{0}(\beta)=\frac{1}{4} b^{T} D^{-1} b+Z(\beta),
$$

where $X$ is a real upper triangular matrix with positive diagonal elements satisfying $X^{T} X=D$ and $Y=-\frac{1}{2}\left(X^{T}\right)^{-1} b$.

Hence, minimizing $Z_{0}$ is equivalent to minimizing $Z(\beta)$. Moreover, when $b=0$, we have

$$
Z_{0}(\beta)=Z(\beta)=(Y-X \beta)^{T}(Y-X \beta) .
$$

Let us consider the least squares problem similar to (9) where all the constraints are in the form of equality, i.e $A \beta=C$. Using the Lagrangian method, we obtain the corresponding normal equation:

$$
\begin{aligned}
A \beta & =C \\
A^{T} \lambda & +X^{T} X \beta=X^{T} Y .
\end{aligned}
$$

Theorem 2: Let $\hat{\beta}^{*}$ be the solution of (11) and $\hat{\beta}_{0}$ be the solution of linear regression model with no constraints. Then, the relationship between $\hat{\beta}^{*}$ and $\hat{\beta}_{0}$ is:

$$
\hat{\beta}^{*}=\left[I-\left(X^{X}\right)^{-1} A^{T} H^{-1} A\right] \hat{\beta}_{0}+\left(X^{T} X\right)^{-1} A^{T} H^{-1} C
$$

where $H=A\left(X^{T} X\right)^{-1} A^{T}$ is a hat matrix (Sen 1990).

Based on Theorem 1 and Theorem 2, Wang, Chukova and Lai (2003) developed a stepwise algorithm for reducing and solving QP problem (4) 
with regression analysis. The following example illustrates the algorithm. Example 4.1: Consider

$$
\begin{aligned}
\min Z_{0}= & -2 x_{1}-3 x_{2}+x_{1}^{2}+x_{2}^{2}+x_{1} x_{2} \\
2 x_{1}+2 x_{2} & \leq 2 \\
3 x_{1}-2 x_{2} & \geq 1 \\
x_{i} & \geq 0 \quad i=1,2 .
\end{aligned}
$$

The solution to this QP by using Wolfe's method is found to be $Z^{T}=$ $\left(x_{1}, x_{2}\right)=\left(\frac{3}{4}, \frac{5}{8}\right)$. Let us apply our algorithm for reducing the above QP to LS. We have

$$
\begin{aligned}
& \min Z_{0}=\left(\begin{array}{ll}
-2 & -3
\end{array}\right)\left(\begin{array}{l}
x_{1} \\
x_{2}
\end{array}\right)+\left(\begin{array}{ll}
x_{1} & x_{2}
\end{array}\right)\left(\begin{array}{ll}
1 & \frac{1}{2} \\
\frac{1}{2} & 1
\end{array}\right)\left(\begin{array}{l}
x_{1} \\
x_{2}
\end{array}\right) \\
& \text { subject to }\left(\begin{array}{rr}
-1 & -2 \\
3 & -2
\end{array}\right)\left(\begin{array}{l}
x_{1} \\
x_{2}
\end{array}\right) \geq\left(\begin{array}{r}
-2 \\
1
\end{array}\right) \\
& \text { and } \quad x_{i} \geq 0, i=1,2 .
\end{aligned}
$$

The above is a matrix representation of model (4). Let

$$
A=\left(\begin{array}{rr}
-1 & -2 \\
3 & -2
\end{array}\right)=\left(\begin{array}{c}
a_{1}^{T} \\
a_{2}^{T}
\end{array}\right), C=\left(\begin{array}{r}
-2 \\
1
\end{array}\right)
$$

and

$$
D=\left(\begin{array}{cc}
1 & \frac{1}{2} \\
\frac{1}{2} & 1
\end{array}\right), b=\left(\begin{array}{l}
-2 \\
-3
\end{array}\right) .
$$

1. Find the matrices $X$ and $Y$ and convert a QP problem to a LS problem.

$$
X=\operatorname{Choleski}(D)=\left(\begin{array}{rr}
1 & \frac{1}{2} \\
0 & .866
\end{array}\right)
$$

and

$$
Y=-\frac{1}{2}\left(X^{T}\right)^{-1} b=(1,1.1547)^{T} .
$$

2. Solve LS $\min Q(\beta)=(Y-X \beta)^{T}(Y-X \beta)$ over $R_{+}^{2}$.

The solution is $\beta^{*}=\left(\frac{1}{3}, \frac{4}{3}\right)^{T}$. 
3. Verify whether $A \beta^{*} \geq C$. In this case both conditions in model (4) are not satisfied. Thus we have to solve the following two problems:

- $\quad$ First, we solve

$$
\begin{array}{rll}
\min & Q\left(\beta^{(1)}\right) & =\left(Y-X \beta^{(1)}\right)^{T}\left(Y-X \beta^{(1)}\right) \\
\text { subject to } & -\beta_{1}^{(1)}-2 \beta_{2}^{(1)} & =-2 \\
& \beta_{i}^{(1)} & \geq 0, i=1,2 .
\end{array}
$$

and obtain

$$
\beta^{(1)}=\left(\frac{1}{3}, \frac{5}{6}\right)^{T}
$$

- Then solve

$$
\begin{array}{rll}
\min & Q\left(\beta^{(2)}\right) & =\left(Y-X \beta^{(2)}\right)^{T}\left(Y-X \beta^{(2)}\right) \\
\text { subject to } & 3 \beta_{1}^{(2)}-2 \beta_{2}^{(2)} & =1 \\
& \beta_{i}^{(2)} & \geq 0, i=1,2 .
\end{array}
$$

and obtain

$$
\beta^{(2)}=(.894739, .8421085)^{T} .
$$

4. Verify whether $A \beta^{(1)} \geq C$ or $A \beta^{(2)} \geq C$.

It is easy to check that the constraint $A \beta^{(i)} \geq C$, for $i=1,2$ is not satisfied. Hence, we solve for

$$
\begin{aligned}
\min & Q\left(\beta^{(1,2)}\right) \\
\text { subject to }-\beta_{1}^{(1,2)}-2 \beta_{2}^{(1,2)} & =-2 \\
3 \beta_{1}^{(1,2)}-2 \beta_{2}^{(1,2)} & =1 \\
\beta_{i}^{(1,2)} & \geq 0, \quad i=1,2,
\end{aligned}
$$

which gives

$$
\beta^{(1,2)}=\left(\frac{3}{4}, \frac{5}{8}\right)^{T}
$$

5. Verify whether $A \beta^{(1,2)} \geq C$.

The constraint $A \beta^{(1,2)} \geq C$ is satisfied. Thus, the the optimal solution is

$$
\hat{\beta}=\beta^{(1,2)}=\left(\frac{3}{4}, \frac{5}{8}\right)^{T} .
$$

The above solution confirms the previous result obtained by Wolfe's method. 


\section{Conclusions}

A linear regression model is solved by two-phase version of the simplex method. A statistical algorithm to solve the quadratic programming problem is proposed. In comparison with the nonlinear programming methods for solving QP, our algorithm has the following advantages:

(a) Statistical courses often form the core portion for most degree programs at bachelor level. The algorithm based on basic statistical concepts is easy to understand, learn and apply.

(b) Some of the steps of the algorithm are included as built-in functions or procedures in many of the commonly used software packages like MAPLE, MATHEMATICA and so on.

(c) The algorithm avoids the usage of slack and artificial variables.

\section{References}

1. W. Kaplan. Maximum and minima with applications, practical optimization and duality. John Wiley and Sons Ltd, 1999.

2. C. L. Lawson and R. J. Hanson. Solving least squares problems. John Wiley and Sons Ltd, 1974.

3. A. C. Rencher. Linear models in statistics. Wiley-Interscience Publications, John Wiley and Sons Ltd, 2000.

4. A. N. Sadovski. L1-norm fit of a straight line: algorithm AS74. Applied Statistics, 23: $244-248,1974$.

5. A. Sen and M. Srivastava. Regression Analysis. Spring - Verlag, 1990.

6. D. Q. Wang, S. Chukova and C. D. Lai. Reducing quadratic programming problem to regression analysis: stepwise algorithm. To appear in European Journal of Operational Research, 2003.

7. P. Whittle. Optimization under constraints. Wiley-Interscience Publications, John Wiley and Sons Ltd, 1971.

8. W. L. Winston. Operations research, with applications and algorithms. Duxbury Press, 1994. 


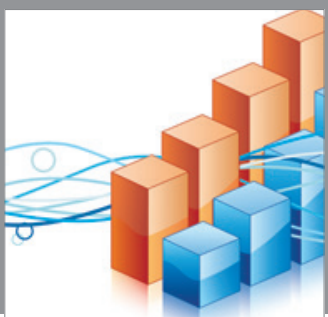

Advances in

Operations Research

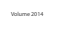

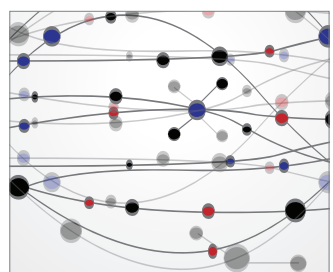

\section{The Scientific} World Journal
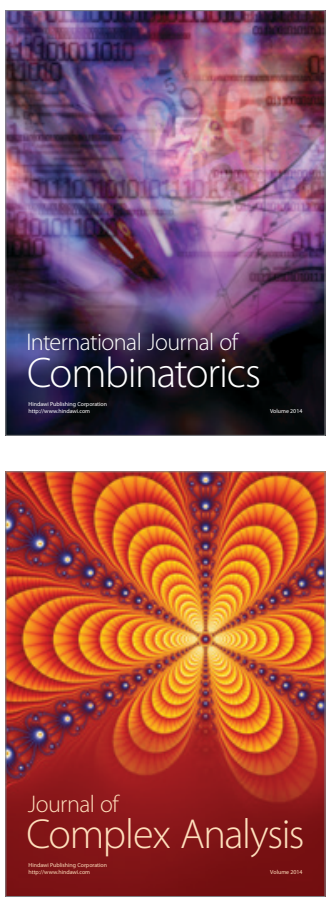

International Journal of

Mathematics and

Mathematical

Sciences
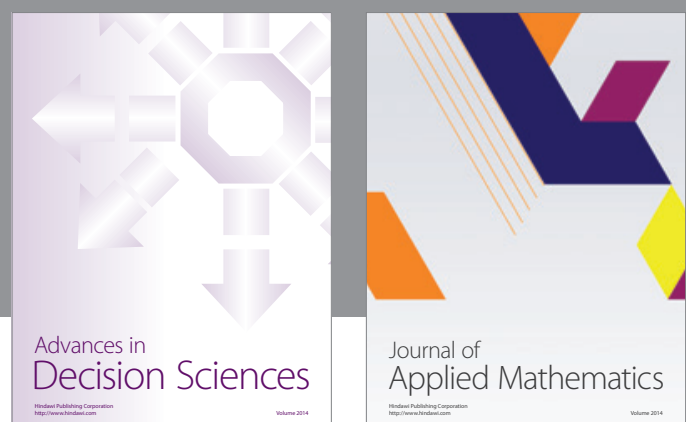

Journal of

Applied Mathematics
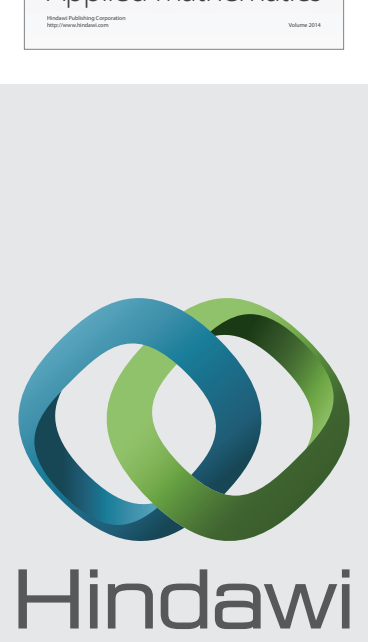

Submit your manuscripts at http://www.hindawi.com
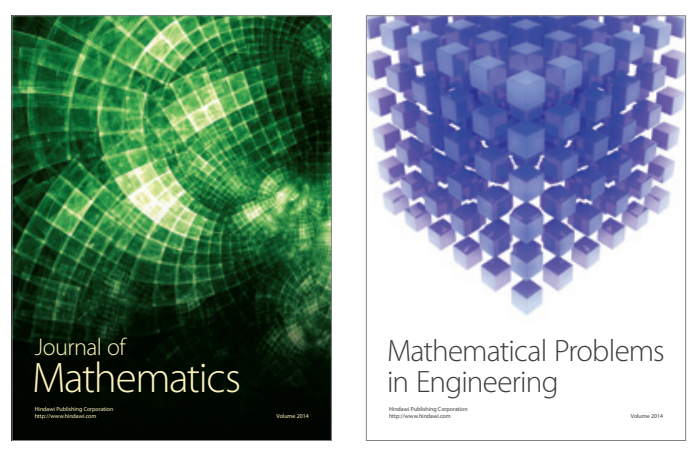

Mathematical Problems in Engineering
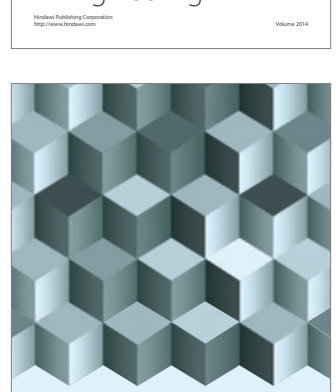

Journal of

Function Spaces
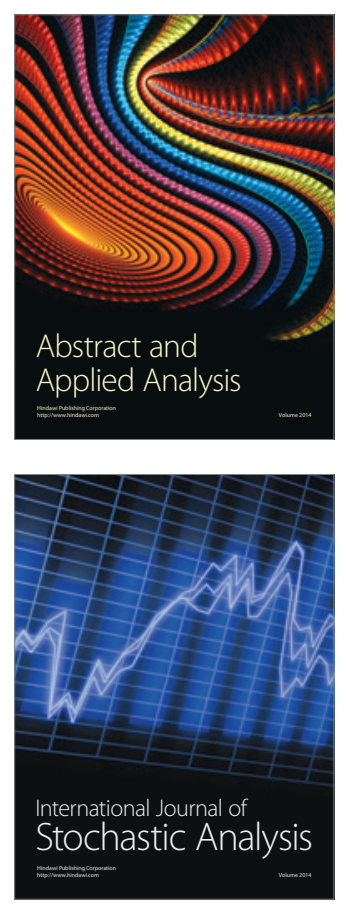

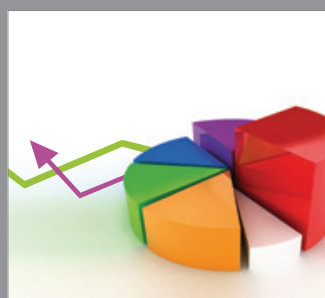

ournal of

Probability and Statistics

Promensencen
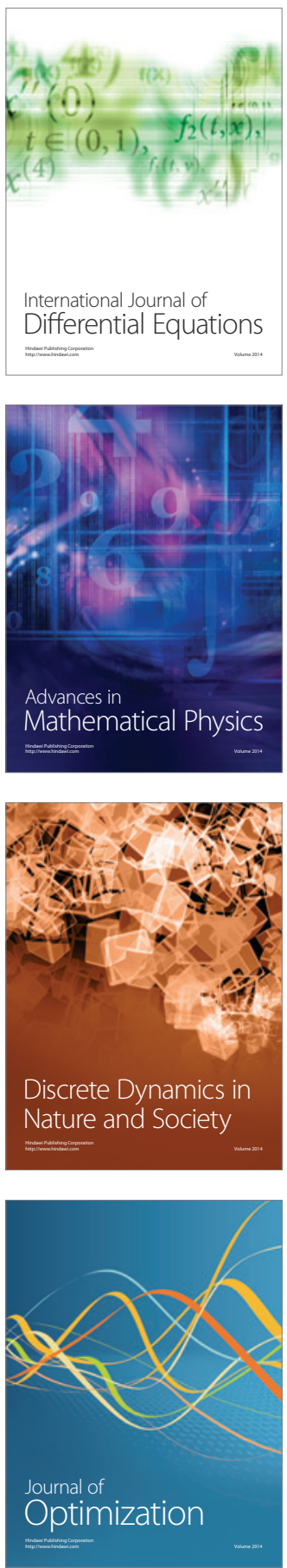